\title{
Concordance and Reproducibility of a Next Generation mtGenome Sequencing Method for High- Quality Samples using the Illumina MiSeq
}

\author{
Michelle A. Peck ${ }^{\mathrm{a}, \mathrm{b}, *}$, Michael D. Brandhagen ${ }^{\mathrm{c}}$, Charla Marshall ${ }^{\mathrm{a}, \mathrm{b}}$, Toni M. Diegoli ${ }^{\mathrm{a}, \mathrm{b}, 1,2}$, Jodi A. \\ Irwin $^{c}$, and Kimberly Sturk-Andreaggi ${ }^{a, b}$
}

\author{
${ }^{a}$ Armed Forces DNA Identification Laboratory, 115 Purple Heart Drive, Dover AFB, DE 19902, United \\ States \\ ${ }^{b}$ American Registry of Pathology Sciences, 9210 Corporate Boulevard, Suite 120, Rockville, MD 20850, \\ United States \\ ${ }^{\mathrm{c}}$ Federal Bureau of Investigation Laboratory, 2501 Investigation Parkway, Quantico, VA 22135, United \\ States \\ ${ }^{1}$ Present address: Defense Forensic Science Center, 4930 N. 31st Street, Forest Park, GA 30297, United \\ States \\ ${ }^{2}$ Present address: ANSER, 5275 Leesburg Pike, Suite N-5000, Falls Church, VA 22041, United States \\ *Corresponding author at: Armed Forces DNA Identification Laboratory, 115 Purple Heart Dr., Dover \\ AFB, DE 19902, United States. Tel.: +1 302346 8519. E-mail address: michelle.a.peck3.ctr@mail.mil
}

\begin{abstract}
Sanger-type sequencing (STS) of mitochondrial DNA (mtDNA), specifically the control region (CR), is routinely employed in forensics in human identification and missing persons scenarios. Yet nextgeneration sequencing (NGS) has the potential to overcome some of the major limitations of STS processing, permitting reasonable paths forward for full mitochondrial genome (mtGenome) sequencing, while also offering higher-throughput and higher sensitivity capabilities. To establish the accuracy and reproducibility of NGS for the development of mtDNA data, 90 DNA extracts that were previously used to generate forensic quality full mtGenomes using STS were sequenced using Nextera XT library preparation and the Illumina MiSeq. Using the same amplicon product, replicate library sets were generated and sequenced at different laboratories, and analysis was performed in replicate using the CLC Genomics Workbench. Both sequencing sets resulted in $99.998 \%$ of positions with greater than 10X coverage when 96 samples (including controls) were multiplexed. Overall, 99.9996\% concordance was observed between the NGS data and the STS data for the full mtGenome. The only "discordant" calls involved low level point heteroplasmies, with the differences resulting from stochastic variation and/or the increased sensitivity of NGS. Higher sensitivity also allowed for the detection of a mixed sample previously not detected with STS. Additionally, variant calls were reproducible between sequencing sets and between software analysis versions with the variant frequency only differing by $0.23 \%$ and $0.01 \%$, respectively. Further validation studies and specialized software functionality tailored to forensic practice should facilitate the incorporation of NGS processing into standard casework applications. The data herein comprise the largest, and likely most thoroughly examined, complete mtGenome STS-NGS concordance dataset available.
\end{abstract}

\section{Keywords}

Mitochondrial genome (mtGenome), Next Generation Sequencing (NGS), Illumina MiSeq, Concordance, Forensics 


\section{Introduction}

Over the past 20 years, Sanger-type sequencing (STS) has been the standard for the analysis of mitochondrial DNA (mtDNA) in forensic casework [1-7]. The high copy number of mtDNA within the cell compared to the nuclear genome and its maternal inheritance make mtDNA analysis advantageous when samples contain low amounts of nuclear DNA (nDNA) and/or direct references are not available for comparison. While forensic mtDNA analysis has traditionally focused on the control region (CR), increased discrimination can be achieved when the full mitochondrial genome (mtGenome) is utilized [813]. STS processing, while effective, is a tedious, costly, and time-consuming process, particularly when analyzing the full mtGenome.

The forensic community has begun to embrace next generation sequencing (NGS) technology as a means to generate large quantities of sequence data for nDNA short tandem repeats (STRs), nDNA single nucleotide polymorphisms (SNPs), and mtDNA [13-22]. One particular challenge to this transition is the lack of legacy sequence data to evaluate concordance, as only length-based data is available for STRs and SNPs have not traditionally been analyzed in forensics. However, because the mtDNA CR is currently the only locus routinely sequenced in forensic laboratories, mtDNA data serve as a particularly good measure of NGS technology since traditional mtDNA concordance data are available for comparison.

To date most mtDNA concordance studies performed with forensic applications in mind have either involved only CR STS data or a small sample set of STS-generated full mtGenome haplotypes due to the availability of the former and demand of generating the latter [13,14,17,23]. Parson et al. performed a larger scale evaluation of sequencing performed on the Ion Torrent Personal Genome Machine (Thermo Fisher Scientific, Waltham, MA) [15], but a similar analysis has not been conducted with the Illumina MiSeq.

Building upon other published methods for sequencing the mtGenome using long range PCR combined with the Nextera XT DNA Sample Preparation Kit (Illumina, San Diego, CA) and the Illumina MiSeq [13,14], as well as internal testing and optimization, a processing and analysis workflow using the CLC Genomics Workbench (CLC bio, Aarhus, Denmark) was developed. This method was used to sequence 90 serum extracts for which forensic-quality STS data were available for the full mtGenome $[9,24,25]$. In addition to assessing concordance with STS profiles, the present study evaluated reproducibility through replicate library preparation and sequencing of the DNA libraries at different laboratories as well as replicate analysis by two scientists. The data herein comprise the largest, and likely most thoroughly examined, complete mtGenome STS-NGS concordance dataset available.

\section{Materials and Methods}

\subsection{Samples}

Ninety DNA extracts were selected from a larger set of anonymized blood serum specimens from the Department of Defense Serum Repository. These DNA extracts were previously processed with STS to generate full mtGenome haplotypes [9,24]. Samples for this study were chosen based on U.S. population group (African American, U.S. Caucasian, and U.S. Hispanic), haplogroup, and unique sequence features (e.g. point heteroplasmy (PHP)). Appropriate controls were also processed, including extraction reagent blanks (RBs), target enrichment positive (PC) and negative (NC) controls, and a library $\mathrm{NC}$, for a total of 96 samples. 
This research was reviewed and approved by the U.S. Army Medical Research and Materiel Command's Office of Research Protections, Institutional Review Board Office.

\subsection{Target enrichment with long range PCR}

Amplification of the 95 samples (90 serum extracts, 3 RBs, target enrichment PC and NC) was performed at the Armed Forces DNA Identification Laboratory (AFDIL). PCR was carried out using previously published primers [26,27] that amplify the full mtGenome with two overlapping amplicons, each approximately 8500 bp in size. The $25-\mu \mathrm{L}$ amplification reactions included $1 \mathrm{X}$ Advantage GC-Melt LA Buffer (Clontech, Mountain View, CA), 0.4 mM dNTPs (Applied Biosystems, Foster City, CA), 0.4 $\mu \mathrm{M}$ primers (Integrated DNA Technologies Inc., Coralville, Iowa), 1.25 U Advantage GC Genomic LA Polymerase Mix (Clontech), and $5 \mu \mathrm{L}$ DNA template. Thermal cycling was performed on a Veriti Thermal Cycler (Applied Biosystems) according to previously published parameters [27]. No postamplification purification was performed.

Amplification success was assessed with the QIAxcel High Resolution Cartridge on the QIAxcel Advanced instrument (QIAGEN, Valencia, CA). A subset of samples ( $\mathrm{n}=14$ for Amplicon A, $\mathrm{n}=17$ for Amplicon B) was also evaluated using the Agilent DNA 12000 Kit (Agilent Technologies, Santa Clara, CA) on the 2100 Bioanalyzer instrument (Agilent Technologies) to generate quantitative data. From these two quantification measures, the average concentration of each amplicon was estimated. Based on this estimated average and a target concentration of $0.2 \mathrm{ng} / \mu \mathrm{L}$, a single dilution factor for each amplicon was applied to all samples regardless of individual concentration, using $10 \mathrm{mM}$ Tris- $\mathrm{HCl}(\mathrm{pH} 8.5)$ for dilution. The two amplicons for each sample were then pooled together in a 1:1 ratio prior to input $(5 \mu \mathrm{L})$ into the Nextera XT DNA reaction.

\subsection{Nextera XT library preparation}

Two 96-sample library pools were generated from the same amplicon input using the Nextera XT DNA Sample Preparation Kit according to the manufacturer's protocol unless otherwise noted $[28,29]$. For each set a library $\mathrm{NC}$ was introduced at the start of the processing. Immediately following target enrichment, Set 1 was generated at the AFDIL with thermal cycling on a TRobot (Biometra, Goettingen, Germany). Library normalization followed a concentration-based approach using the KAPA Library Quantification Kit for Illumina Platforms (Kapa Biosystems, Wilmington, MA) on the 7500 Real-Time PCR System (Applied Biosystems) to quantify libraries [30]. Based on these concentrations, samples were normalized to $2 \mathrm{nM}$ by individually diluting with $10 \mathrm{mM}$ Tris- $\mathrm{HCl}(\mathrm{pH} 8.5)$. Dilution was not performed if the sample or control was below $2 \mathrm{nM}$. The 96 normalized libraries were then combined (5 $\mu \mathrm{L}$ per library) to create the Set 1 pool. The amplification products were stored for 3 months at $4{ }^{\circ} \mathrm{C}$ prior to generating the second library (Set 2) at the Federal Bureau of Investigation Laboratory (FBI Laboratory). Thermal cycling was performed on a GeneAmp PCR System 9700 (Applied Biosystems). Library normalization and pooling were performed according to the Nextera XT DNA Preparation Kit using the bead-based strategy. A subset of samples from both libraries were evaluated with the Agilent High Sensitivity (HS) DNA Kit (Agilent Technologies) on the 2100 Bioanalyzer to confirm library quality prior to normalization and pooling.

\subsection{Sequencing}

Sequencing was performed on the Illumina MiSeq using the MiSeq Reagent v3 600-cycle kit (Illumina) according to the manufacturer's recommendations for paired end sequencing $(2 \times 300$ cycles $)$ 
[31]. Set 1 was loaded at a final concentration of $10 \mathrm{pM}$ and sequenced on a MiSeq located at the AFDIL. Set 2 was prepared for sequencing according to the manufacturer's recommendations for beadbased normalized libraries and sequenced on a MiSeq located at the FBI Laboratory [29].

\subsection{Data analysis}

MiSeq Reporter (Illumina) generated demultiplexed FASTQ files from the raw data, and the paired reads from both data sets were imported into the CLC Genomics Workbench (v7.0.3) for analysis at the AFDIL. The following workflow was developed for sequence alignment: 1) sequences were trimmed $20 \mathrm{bp}$ on both the 5' and 3' ends, 2) sequences <100 bp were discarded, 3) overlapping pairs were merged, and 4) both merged and unmerged pairs were mapped to the revised Cambridge Reference Sequence (rCRS) [32,33]. This workflow was optimized based on these sequencing sets and previous positive control testing to eliminate adapter dimers, remove target enrichment primer sequences, minimize the impact of errors at the ends of reads, improve read quality, and make full use of the available reads.

Variant calling was performed using the quality-based variant detection tool and required a minimum of $10 \mathrm{X}$ coverage. At positions that exceeded this coverage threshold, variants were reported if the variant frequency (VF) was $\geq 5 \%$ and the read count supporting the variant was $\geq 10$. For example, a variant called at $4 / 43$ (variant count/coverage) would not be reported. A variant position was considered homoplasmic if the VF was above $95 \%$. If the $\mathrm{VF}$ was $\leq 95 \%$ and a second nucleotide was detected at $\geq 5 \%$ with a variant count of 10 , the variant was defined as a point heteroplasmy (PHP) and the appropriate International Union of Pure and Applied Chemistry code was used to represent the mixed position. A lowercase nucleotide base was used to indicate the presence of an insertion/or deletion (indel) in conjunction with a nucleotide (variant or reference base). Due to known alignment issues with indels, the major molecule was reported ( $\mathrm{VF} \geq 50 \%$ ). Position 3107, while not a true variant, was included in data analysis to further assess indel alignment issues and to provide a measure of sequencing quality control as every sample should contain the $3107 \mathrm{del}$ call; however, in practice the $3107 \mathrm{del}$ would not be reported in the final haplotype [34]. To further evaluate low level variants present at frequencies below $5 \%$, additional analyses were performed with a $1 \% \mathrm{VF}$ threshold. Results (e.g. reported coverage, VF metrics) generated using CLC Genomics Workbench v7.0.3 were utilized for in-depth data analysis and comparison. Data sets were also analyzed in the same manner at the FBI Laboratory using a newer version of CLC Genomics Workbench (v7.5.1) and these results were utilized to confirm variant calling and evaluate similar performance of the software versions.

To assess concordance, the CLC-generated haplotypes (5\% threshold) for Set 1 were compared to the STS haplotype for each sample. The STS haplotypes represent data developed following a multi-step review process [9]. Independent reviewers checked for any differences between the STS and NGS haplotypes. A call was classified as discordant if the variant was observed in one data type but not in the other, or the variant was called differently between the two sequencing methods (e.g. T16263C vs. T16263Y). The CLC Workbench employs a 5' indel alignment whereas forensic nomenclature guidelines recommend a 3' alignment [2,6,7,15]. However, instances of nomenclature differences were not considered discordant as long as the string sequence was consistent (e.g. A4315del and A4317del). Further, due to the complexity of analyzing length heteroplasmy (LHP) present in NGS data, the following regions were reviewed but not considered in the concordance assessment: 303-315 (hypervariable (HV) II region), 513-524 (AC-repeat region), 568-573 (HVIII region), 956-965, 58935899, 8270-8289, 12418-12425, and 16180-16193 (HVI region). Once analysis of Set 1 haplotypes was 
complete, Set 2 haplotypes were used to assess reproducibility. Finally, the AFDIL (v7.0.3) and FBI (v7.5.1) data reviews were then cross-compared for each set to confirm the analysis. Discordant (NGS vs. STS) or inconsistent (NGS vs. NGS) calls were then investigated further to determine the cause of discrepancy. After this additional analyst review, a final concordance value was calculated.

\section{Results and Discussion}

\subsection{Library preparation and sequencing}

Target enrichment of the entire mtGenome was carried out on 95 samples generating two amplicons of approximately $8500 \mathrm{bp}$ each. All samples produced sufficient amplified product of the expected target size for both amplicons; no amplification product was detected for any of the negative controls (RBs, target enrichment NC). Using both QIAxcel and Bioanalyzer results, estimated average PCR product yields were $211.95 \mathrm{ng} / \mu \mathrm{L}$ for Amplicon A and $161.65 \mathrm{ng} / \mu \mathrm{L}$ for Amplicon B. In order to streamline the simultaneous processing of 96 samples, amplicons were not individually diluted to the target $0.2 \mathrm{ng} / \mu \mathrm{L}$ but rather diluted using a single dilution factor (approximately 1000-fold) based on the average concentration of each amplicon. Using a $5 \mu \mathrm{L}$ input volume resulted in a range of DNA inputs for the Nextera XT reaction. The maximum input was approximately $3 \mathrm{ng}$, while the least concentrated sample (mtGAfrLA0006) had approximately $100 \mathrm{pg}$ of DNA input, which is ten-fold less than the $1 \mathrm{ng}$ input recommended by the manufacturer [28].

Despite these differences in input concentrations, two sets of libraries were successfully generated using the Nextera XT method. Set 1 normalization was performed with the KAPA qPCR method instead of the manufacturer's recommendation of the Bioanalyzer HS Kit (11 samples/chip) because of its higher throughput capacity (up to 90 samples/run). The average KAPA qPCR concentration of the Set 1 purified libraries was $6.47 \mathrm{nM}$. The concentrations of all RBs and NCs were less than $0.02 \mathrm{nM}$. In addition, only two samples were below the $2 \mathrm{nM}$ concentration recommended for sequencing (mtGHispCA0004 - $1.34 \mathrm{nM}$ and mtGHispCA0006 - $1.89 \mathrm{nM}$ ) and thus were not diluted [28]. A subset of samples $(n=9)$ checked on the Bioanalyzer prior to normalization resulted in an average concentration of $8.8 \mathrm{nM}$. Set 2 was prepared using bead-based normalization, which does not result in quantification results. For reference, a subset of these samples $(n=4)$ was analyzed on the Bioanalyzer prior to normalization and averaged a concentration of $9.5 \mathrm{nM}$.

The two libraries were sequenced on the respective MiSeq instruments and minimal differences were seen between the two runs in terms of sequencing quality metrics (Table 1). Both runs had over 900 $\mathrm{k} / \mathrm{mm}^{2}$ cluster density with about $90 \%$ of clusters passing filter. However, while both runs yielded approximately 20 million reads passing filter, the distribution of reads per sample (read percentage) was more variable in Set 2 than in Set 1 (Figure 1). With 96 samples, approximately 1\% of the final data would be expected for each sample and, indeed, the median read percentage of run data per sample was near $1 \%$ in both runs. However, Set 2 showed a wider range of values $(0.04-1.63 \%)$ with 10 samples falling below $0.5 \%$ of the total reads, compared to Set $1(0.52-1.63 \%)$. Even the two samples in Set 1 that were below the recommended $2 \mathrm{nM}$ library input did not show notably reduced read percentage.

The different normalization strategies used to generate each library are likely the cause of the read percentage differences, as similar patterns were observed in other comparisons of these two libraries (data not shown). The bead-based normalization method is likely more inconsistent due to stochastic variation in bead concentration per sample, the multiple sample manipulation steps, and challenging manual bead washing steps. Yet even the samples with lower read percentages, and subsequently lower average coverage, had sufficient coverage for accurate variant calling. In the end, libraries were 
successfully generated with the Nextera XT method using either normalization strategy from a wide range of DNA inputs (100 pg $-3 \mathrm{ng}$ ).

\subsection{Concordance and reproducibility}

Using previously developed STS haplotypes as the basis for comparison, this sequencing strategy generated concordant profiles across the full mtGenome, confirmed by replicate processing and analysis. Of the approximately 1.5 million bases (16,479 positions in 90 samples) examined in Set 1,3309 substitutions, 114 indels, and 25 expected PHPs were concordant with the STS data. Only 42 of the 3485 variant positions were initially identified as differences from the STS data, equating to $99.997 \%$ concordance across 1,483,110 analyzed positions (Tables 2 and 3). The vast majority of these variants were reproduced in Set 2 (Table 3). The calls that were not consistent between the two NGS sets were calls that were also discordant with STS. All differences were reviewed further and separated into four categories: below the coverage threshold, alignment artifact, mixture, and low level variants (Table 3). All variant calls were consistent between the v7.0.3 and v7.5.1 analysis.

\subsubsection{Coverage}

Both data sets resulted in high sample average coverage at $2139 \mathrm{X} \pm 400$ and $1676 \mathrm{X} \pm 606$ across the analyzed positions of the mtGenome, respectively. All samples in Set 1 had average coverage greater than 1000X, while 11 samples in Set 2 were below 1000X coverage. Consistent with lower read percentage per sample, samples mtGAfrLA0006 and mtGCaucMA0006 in Set 2 had less than 200X average coverage. Though average coverage provides some measure of data quality, the reliability of any given base call is dependent on read coverage at that particular position. On a site-by-site basis, $99.998 \%$ of positions in both NGS data sets exceeded the 10X coverage threshold used for variant calling despite the differences in average coverage per sample.

The distribution of coverage across the mtGenome was consistent across both sets of data and all haplotypes. Coverage did not seem to be affected by a specific haplotype or haplogroup, as 90 unique haplotypes and 81 different haplogroups were included in this study [9], and coverage patterns were consistent across all of them (Figure 2). Instead, the observed pattern of coverage was consistent with what has been described in previous studies, in which coverage was reduced at the HVII polycytosine stretch and around np 3500 when using Nextera XT library preparation [13,14,16,35]. The dip at HVII is likely a result of a combination of sequencing difficulty in this region and complex alignment of homopolymeric regions resulting in fewer aligned reads. Vancampenhout et al. reported the decrease in coverage near np 3500 could be the result of the transposon utilized in Nextera XT preferentially targeting this region [35], though the feature has also been observed in mechanically sheared datasets [36].

Only one sample in each set (Set $1-23$ positions in mtGCaucMN0003, Set $2-32$ positions in mtGAfrLA0006) contained regions below 10X coverage. Overall, these areas comprised less than $0.2 \%$ of each mtGenome and were near or within the dips known to occur (e.g. LHP regions, np 3500 region). Though regions such as these that fall below the established coverage threshold would generally not be considered in forensic practice, the low coverage regions were considered here for informational purposes and for Set 2 mtGAfrLA0006 in particular. In Set 1 this sample produced a haplotype 100\% concordant with the STS data. However, in Set 2 mtGAfrLA0006 had the lowest average coverage (90X) across all samples and the haplotype lacked the C3594T variant. This was the only instance in which a variant (as opposed to a PHP) was not reproducible and analyst review confirmed that the low coverage was the 
cause of the difference. In the final assessment, the positions below 10X were excluded as they were below the detection threshold.

This example highlights the necessity of reporting the range of positions that exceed the coverage threshold and for which variant calls can be made, as is currently done for STS data but not readily available in most NGS analysis software. Without this information in any given output file, no differentiation is made between a position consistent with the reference and a position not passing the coverage threshold. While coverage information can be obtained from different outputs or visual inspection of the alignment, automatic output of the reportable range would streamline analysis. Overall, the tested data generation and analysis methods resulted in very few positions below the coverage threshold. However when such data are encountered, software functionality that specifies the interpreted range would facilitate analysis moving forward.

\subsubsection{Substitutions and indels}

Frequencies of homoplasmic substitution and indel variants were highly consistent within and between data sets. The substitution VF averaged $99.65 \% \pm 0.56$ in Set 1 and $99.64 \% \pm 0.58$ in Set 2, despite the differences in coverage observed (Table 4). Indels, however, were observed at lower and more variable VF, ranging from $81.82-99.51 \%$ in Set 1 and $87.89-99.17 \%$ in Set 2. This lower VF can largely be attributed to alignment issues when the indel occurs at the end of a sequence read. The correct string sequence was clearly present in these cases, indicating this was not a problem with the chemistry or sequencing instrument. All indels showed similar alignment artifacts, suggesting that changes to the software would likely be required to address this issue.

Based on combined Set 1 and Set 2 data, the 99.7\% confidence interval (average $-3 *$ standard deviation) was determined to be $97.94 \%$ and set as the expected substitution VF threshold. Several substitution positions were identified to have alignment issues similar to indels due to the fact that the VFs routinely fell below this threshold. The most severe artifact was observed when the T10873C variant was present, which results in a stretch of $5 \mathrm{Cs}$. When the variant was at the end of the read, the software favored a deletion. As a result, the average VF at this position only averaged $96.20 \% \pm 0.91$ (39 samples per set). The deletion was observed above the 5\% threshold in six samples (Set 1 - four samples, Set 2 two samples) resulting in the T10873c call. While these were noted as discordant calls for the initial assessment and accounted for 6 of the NGS set inconsistencies, analyst review established that the raw data are in fact concordant between NGS and STS and thus was excluded from the final concordance value. Additionally, several other positions (np 456, 9477, 9545, 12414, 16362) that routinely exhibited VF below the expected value of $97.94 \%$ were identified to be the result of deletion artifacts occurring at the end of reads near homopolymeric stretches (Supplemental Table 1). Optimization of software packages and read alignment algorithms to accommodate specific sequence features are likely to correct these types of bioinformatic artifacts. Indeed, some preliminary testing at the AFDIL of a modified mtDNA analysis workflow that employs local realignment steps improves gap alignment and eliminates the artificial deletion calls (data not shown).

To further assess the reproducibility of variant calling between different library preparations and sequencing sets, VFs were compared directly between the two NGS data sets and averaged a difference of only $0.23 \% \pm 0.39$. Substitution VFs (excluding np 10873) were highly similar with an average difference of only $0.17 \% \pm 0.21$ between sets. More variability was observed for indels $(0.83 \% \pm 1.07)$ due to known alignment artifacts whereas stochastic variation of low level variants caused higher VF differences for PHPs $(0.99 \% \pm 1.20)$. Substitution positions that exhibited the highest VF difference 
between sets were characterized by low coverage in at least one of the data sets. The 33 positions that exhibited greater than $1.00 \%$ difference between the two sets averaged $434 \mathrm{X}$ coverage in the sample with the lower coverage (Supplemental Table 2), while all other positions averaged 1376X coverage. The greatest difference was observed at np 3594 in mtGAfrFL0008, with VF of 95.45\% in Set 1 (264X) and $98.06 \%$ in Set $2(310 \mathrm{X})$. Authentic low level variants (i.e. PHPs) are potentially a contributing factor to this variability. However, the greater VF difference at the lower coverage positions is more likely due to the fact that background signal has a greater impact on VF when coverage is low. Though these finescale differences could be detected, their magnitude was small. Overall, highly similar VF values were observed on a position by position, sample-by-sample and run-by-run basis, underscoring the robustness and reproducibility of the process.

\subsubsection{PHPs}

The majority of the STS-confirmed PHPs were reproducibly called in each NGS set (Set 1 25/26, Set 2 - 24/26), and the VFs were consistent with the expected abundance based on STS estimates (Supplemental Table 3). As noted earlier, there was greater variation in the PHP VF differences between the two NGS sets, most likely as a result of stochastic variation at various steps of the library preparation and sequencing process. Stochastic variation is also likely contributing to the two PHPs that were expected from the STS data but not called in the NGS data. First, the A189R PHP in mtGCaucPA0010 was correctly called in Set 1 (7.19\%, 59/821 reads), but was just below the user-defined 5\% threshold in Set 2 (4.86\%, 32/658 reads). In the other instance, the G143R PHP in sample mtGAfrFL0008 was not called in either set because it was well below the VF threshold (1.37\% and $1.51 \%$, respectively). Although it is unusual that such a low level PHP would be identified with STS data, high data quality with minimal background allowed for visual detection of this approximately 5\% STS variant by the analysts (Supplemental Figure 1). Additionally, the STS data were generated with a smaller and more sensitive amplicon ( $1200 \mathrm{bp})$ [25] that would accommodate any degraded DNA within the serum sample extracts, in contrast to the long range target ( $\sim 8500 \mathrm{bp})$ used in this study. As a result, the combination of differences in amplicon size and efficiency may have exacerbated stochastic variation in VF. While these PHPs were considered discordant, this fluctuation in VF is not unexpected for low level variants.

Most of the discordant calls initially observed in this study were PHPs called in the NGS data but not in the STS data, which also accounted for the majority of inconsistent NGS calls (Table 3). Further, 32 discordant PHPs in Set 1 and 31 in Set 2 were seen in a single sample (mtGAfrAL0004). Given the high number of PHPs detected in this sample, the sample was more closely investigated and determined

to be a mixture. Although the detection of the mixed positions varied between the two sets due to stochastic variation, the majority $(n=24)$ were consistent. The low level variants were consistent with another sample on the original extract plate, while the dominant profile in the mixture matched the expected haplotype for mtGAfrAL0004. Additional STS was performed with the amplicon product used for library preparation to confirm the mixture did not occur during the NGS library preparation process. Both the original and reprocessed STS data gave some indication of a mixture at certain positions. However, because the minor component peaks were not above the background signal, they were appropriately not identified by the analysts reviewing the STS data. These particular discordances simply highlight the increased ability to detect low level variants due to the sensitivity of NGS technology. Following identification of this sample as a mixture, it was removed from further analyses. 
Another indicator that mtGAfrAL0004 was a mixture was the high number of variant positions that were below the $97.94 \%$ substitution VF threshold. The only three samples that had more than two substitutions below the $97.94 \%$ threshold were mtGAfrAL0004 (Set $1-3$ variants, Set 2 - 5 variants), mtGAfrFL0008 (Set 1 - 12 variants, Set 2 - 13 variants), and mtGAfrMI0006 (Set 1 - 4 variants, Set 2 6 variants) (Supplemental Table 1). These three samples accordingly had the lowest sample substitution VF average in both sets. This data suggests that mtGAfrFL0008 and mtGAfrMI0006 may also be low level mixtures, though below the 5\% threshold used here for variant calling. It is plausible the STSdetected PHP in mtGAfrFL0008 that was not detected in either of the NGS data sets, was from a low level contaminant that did not meet the NGS VF threshold. Given the consistency in VF for single-source samples from run-to-run of even low level variants, VF could potentially be useful as a quality control measure to identify mixtures.

The remaining discrepant PHPs were detected by NGS but not seen in the STS data; all but one was reproduced between NGS sets (Supplemental Table 3). The PHP that was only observed in one of the data sets (Set 2, mtGAfrWI0001 - G9655R) fell just below the threshold in Set 1 (4.94\%, 102/2068 reads). For those PHPs detected with NGS but not STS, the minor component averaged $7.12 \%$ VF. In contrast, the minor components observed for PHPs that were detected in both STS and NGS data averaged 25.65\% VF. The lower VF of the additional "discordant" PHPs indicates they were below the range of detection with STS, but detectable with NGS. The reproducibility of the PHPs and the fact they are consistent with reported patterns for coding region PHPs [37] further support the authenticity of these low level variants.

\subsubsection{Controls}

The positive control, initiated at target enrichment, was fully concordant with previously processed STS data and reproducible between NGS sets, including a PHP at G8078R (Set $1-8.96 \%$, Set $2-9.16 \%$ ). Coverage averaged $1390 \mathrm{X}$ and $1803 \mathrm{X}$ respectively, with no positions below $10 \mathrm{X}$ across the entire mtGenome. In both data sets, VFs of the different variant types (e.g. substitutions) were consistent with the values reported for all other samples. In contrast, all of the negative controls ( $3 \mathrm{RBs}, 1$ target enrichment NC, and 1 library NC) resulted in less than $0.01 \%$ read percentage and subsequently insufficient coverage $(<10 \mathrm{X})$ across the entire mtGenome. Accordingly, no variants were called. Due to the sensitivity of NGS technology a small number of reads will likely routinely be detected in the NCs, but the abundance will be dependent on the specific processing and analysis strategies. It is essential in future validation studies to characterize signal in the NCs and establish thresholds to classify a control as acceptable.

\subsubsection{Software comparison}

To confirm analysis reproducibility, scientists at the respective laboratories each performed analysis using the same parameters, but two software versions were used due to availability. As noted earlier, variant calls were exactly the same between the v7.0.3 and v7.5.1 versions in each NGS data set. Comparing the VFs and coverage at the variant positions between software versions indicated there was minimal impact on analysis. The VF averaged a difference of only $0.01 \% \pm 0.04$, while the coverage averaged a difference of $3.1 \mathrm{X} \pm 9.2$. Though the two samples with the largest coverage difference had higher coverage in v7.0.3, the majority of samples (95\%) had higher coverage with v7.5.1 (Supplemental Table 4). Additionally, the maximum coverage difference in $98 \%$ of the samples was observed at variant positions near the difficult HVII region, for which v7.5.1 coverage was greater in all instances. The 
increase in coverage is consistent with improved read mapper functionality in v7.5.1 as reported by the manufacturer, though the impact on VF was negligible. While slight differences were observed in these metrics, the variant calls were confirmed by two scientists and attest to the reproducibly of the analysis even with the use of different software versions.

\subsubsection{Final concordance and reproducibility assessment}

The initial concordance assessment identified only 42 discordant positions, equating to $99.997 \%$ concordance. Yet detailed analyst review of the discordant calls resulted in a final concordance value of 99.9996\% (six discordant calls out of 1,466,608 positions in Set 1) based on the following adjustments (Tables 2 and 3). First, due to coverage below 10X, 23 positions were excluded from the calculation. Second, the T10873c call was determined to be consistent with the T10873C STS call by examining the sequence data and was thus considered concordant. Finally, sample mtGAfrAL0004 was excluded entirely due to its classification as a mixture. The remaining six discordant calls all involved PHPs and were either the result of increased sensitivity with NGS or stochastic variation, which also accounts for the two differences between the NGS data sets. Compared to Set 1, Set 2 only differed by two low level variants (excluding the C3594T difference due to insufficient coverage), confirming reproducibility between NGS sets (Table 3). No variant call differences were observed between the two software versions, and only minor variation in analysis metrics. Sequencing the full mtGenome with this method was demonstrated to be concordant with STS data and reproducible at the library preparation, sequencing, and analysis levels.

\section{Conclusion}

This is the first study to demonstrate on a large-scale the concordance of full mtGenomes generated using the Illumina MiSeq with STS data. Library preparation using Nextera XT and Illumina sequencing were shown to be reproducible across laboratories and MiSeq instruments, confirmed by replicate analysis by independent reviewers on different software versions. After final analyst review of the data sets, the 89 NGS-generated haplotypes were $99.9996 \%$ concordant with STS data. The discordant positions all involved low level variants (PHPs) for which either stochastic variation or increased sensitivity of NGS was the cause of the discrepancy. Importantly, no incorrect substitution or indel calls were observed.

The analysis software and workflow described here were adequate for assessing concordance and reproducibility of the laboratory processing and sequencing chemistry. However, the analysis highlighted software adjustments that would facilitate implementation into forensic casework. First, improved alignment strategies that address issues at the ends of reads, particularly in homopolymeric regions, would result in more accurate VF reporting of indels and specific substitutions. While not dealt with specifically in this study, tools that standardize the alignment of LHP would be similarly beneficial. Though these regions have minimal impact on final data interpretation in casework scenarios, LHP variants are reported by some laboratories. Finally, an output that specifies the range of positions above the minimum coverage threshold is necessary. Final profile generation (i.e. alignment/nomenclature adjustments and determination of reportable range) was performed by the analyst in this study, yet automated incorporation of these forensic practices into the software would both ease analysis and help ensure

accurate variant calls. Such software tools are now available to the forensic community to address some of these needs [38,39], and a detailed assessment of these tools is warranted. 
Increased sensitivity is one of the major advantages of NGS and was demonstrated by this study in the detection of the mixed sample and an additional six PHPs that had previously gone undetected with STS. The 5\% VF threshold was selected as a comparison to STS, as this is at the lower limit of detection with pristine STS data [9]. At this level, concordant profiles were obtained. However, as other studies have demonstrated it is likely this detection threshold could be lowered [23,40]. The VF analysis showed that variants are reliably called above $97.94 \%$ VF within and between sequencing sets. In essence, this is a preliminary assessment of the background signal though only looking at variant positions. In order to get the complete picture, every position should be examined to establish the expected level of background signal. Sensitivity and mixture studies are thus needed to establish a detection threshold that ensures low level variants (either authentic to the sample or from a contaminant sample) can be reliably distinguished from background noise.

This analysis indicates that coverage will be a critical parameter in establishing the lower limits of sensitivity. At lower coverage, a few reads (i.e. background signal) will have a greater impact on VF and thus necessitate a higher detection threshold. The dynamic relationship between coverage and VF needs to be established with further studies. In identifying what level of coverage is required, it is important to consider that coverage across the mtGenome will never be perfectly balanced. While different library preparation methods may provide greater balance, regions of reduced coverage will always be the limiting factor in establishing lower limits of detection. Additionally, when processing in high-throughput fashion and developing workflows for operational environments, a balance between sensitivity, efficiency, and cost must be determined. Comprehensive mixture and sensitivity experiments will provide better insight into the lower limit of variant detection thresholds with particular coverage expectations based on laboratory processing (e.g. level of multiplexing). While lower detection thresholds with NGS will be useful for many applications such as the resolution of mixtures, this study has demonstrated that a 5\% VF threshold is suitable for high-throughput processing of 96 high quality samples.

Overall, the fine-scale analyses performed here on a combined NGS/STS dataset of 270 complete mtGenomes (180/90, respectively) further establish the reliability of NGS for forensic applications and bring forensic laboratories one step closer to implementation of this technology into routine mtDNA casework.

\section{Acknowledgments}

The authors would like to thank Erin Gorden, Jennifer Higginbotham, Joseph Ring, Jocelyn Bush, Spence Fast, and Quentin Gauthier (American Registry of Pathology, Armed Forces DNA Identification Laboratory) for laboratory and analysis assistance; James Ross (American Registry of Pathology, Armed Forces DNA Identification Laboratory), Jon Norris (Future Technologies, Inc.), Richard Coughlin and Timmathy Cambridge (Armed Forces Medical Examiner System) for technical assistance; Rebecca Just (American Registry of Pathology, Armed Forces DNA Identification Laboratory) for manuscript review; Timothy McMahon, James Canik, and Cynthia Thomas (American Registry of Pathology), Shairose Lalani, Lanelle Chisholm, Lt Col Garner, Lt Col Briones, and COL Ladd Tremaine (Armed Forces Medical Examiner System) for administrative and logistical support, and Ken Kroupa and the Rapid Reaction Technology Office, ASD (R\&E) for funding. The opinions or assertions presented hereafter are the private views of the authors and should not be construed as official or as reflecting the views of the Department of Defense, its branches, the U.S. Army Medical Research and Materiel Command, the 
Armed Forces Medical Examiner System, the Federal Bureau of Investigation, the Department of Justice, or the U.S. Government. This is FBI Laboratory publication number 16-10.

\section{References}

[1] M.M. Holland, D.L. Fisher, L.G. Mitchell, W.C. Rodriguez, J.J. Canik, C.R. Merril, et al., Mitochondrial DNA sequence analysis of human skeletal remains: Identification of remains from the Vietnam war, J Forensic Sci. 38 (1993) 542-553.

[2] M.R. Wilson, M. Stoneking, M.M. Holland, J.A. DiZinno, B. Budowle, Guidelines for the Use of Mitochondrial DNA Sequencing in Forensic Science, Crime Lab.Digest. 20 (1993) 68-77.

[3] P. Gill, P.L. Ivanov, C. Kimpton, R. Piercy, N. Benson, G. Tully, et al., Identification of the remains of the Romanov family by DNA analysis, Nat.Genet. 6 (1994) 130-135.

[4] R.J. Steighner, M. Holland, Amplification and sequencing of mitochondrial DNA in forensic casework, Methods Mol.Biol. 98 (1998) 213-223.

[5] M.M. Holland, T.J. Parsons, Mitochondrial DNA sequence analysis - validation and use for forensic casework, Forensic Sci Rev. 11 (1999).

[6] A. Carracedo, W. Bar, P. Lincoln, W. Mayr, N. Morling, B. Olaisen, et al., DNA commission of the international society for forensic genetics: guidelines for mitochondrial DNA typing, Forensic Sci.Int. 110 (2000) 79-85.

[7] W. Parson, L. Gusmao, D.R. Hares, J.A. Irwin, W.R. Mayr, N. Morling, et al., DNA Commission of the International Society for Forensic Genetics: revised and extended guidelines for mitochondrial DNA typing, Forensic.Sci.Int.Genet. 13 (2014) 134-142.

[8] M.D. Coble, R.S. Just, J.E. O'Callaghan, I.H. Letmanyi, C.T. Peterson, J.A. Irwin, et al., Single nucleotide polymorphisms over the entire mtDNA genome that increase the power of forensic testing in Caucasians, Int.J.Legal Med. 118 (2004) 137-146.

[9] R.S. Just, M.K. Scheible, S.A. Fast, K. Sturk-Andreaggi, A.W. Röck, J.M. Bush, et al., Full mtGenome reference data: Development and characterization of 588 forensic-quality haplotypes representing three U.S. populations, Forensic Science International: Genetics. 14 (2015) 141-155.

[10] T.J. Parsons, M.D. Coble, Increasing the forensic discrimination of mitochondrial DNA testing through analysis of the entire mitochondrial DNA genome, Croat.Med.J. 42 (2001) 304-309.

[11] M.D. Coble, P.M. Vallone, R.S. Just, T.M. Diegoli, B.C. Smith, T.J. Parsons, Effective strategies for forensic analysis in the mitochondrial DNA coding region, Int.J.Legal Med. 120 (2006) 27-32.

[12] A. Brandstatter, T.J. Parsons, W. Parson, Rapid screening of mtDNA coding region SNPs for the identification of west European Caucasian haplogroups, Int.J.Legal Med. 117 (2003) 291-298.

[13] J.L. King, B.L. LaRue, N.M. Novroski, M. Stoljarova, S.B. Seo, X. Zeng, et al., High-quality and high-throughput massively parallel sequencing of the human mitochondrial genome using the Illumina MiSeq, Forensic.Sci.Int.Genet. 12 (2014) 128-135. 
[14] J.A. McElhoe, M.M. Holland, K.D. Makova, M.S. Su, I.M. Paul, C.H. Baker, et al., Development and assessment of an optimized next-generation DNA sequencing approach for the mtgenome using the Illumina MiSeq, Forensic.Sci.Int.Genet. 13 (2014) 20-29.

[15] W. Parson, C. Strobl, G. Huber, B. Zimmermann, S.M. Gomes, L. Souto, et al., Evaluation of next generation mtGenome sequencing using the Ion Torrent Personal Genome Machine (PGM), Forensic.Sci.Int.Genet. 7 (2013) 543-549.

[16] W. Parson, G. Huber, L. Moreno, M.B. Madel, M.D. Brandhagen, S. Nagl, et al., Massively parallel sequencing of complete mitochondrial genomes from hair shaft samples, Forensic.Sci.Int.Genet. 15 (2015) 8-15.

[17] M. Mikkelsen, R. Frank-Hansen, A.J. Hansen, N. Morling, Massively parallel pyrosequencing of the mitochondrial genome with the 454 methodology in forensic genetics, Forensic.Sci.Int.Genet. 12 (2014) 30-37.

[18] C. Borsting, N. Morling, Next generation sequencing and its applications in forensic genetics, Forensic Science International: Genetics. http://dx.doi.org/10.1016/j.fsigen.2015.02.002 (2015).

[19] M. Scheible, O. Loreille, R. Just, J. Irwin, Short tandem repeat sequencing on the 454 platform, Forensic Science International: Genetics Supplement Series. 3 (2011) e357-e358.

[20] J. Irwin, R. Just, M. Scheible, O. Loreille, Assessing the potential of next generation sequencing technologies for missing persons identification efforts, Forensic Sci.Int.Genet.Suppl.Ser. 3 (2011) e447e448.

[21] D.M. Bornman, M.E. Hester, J.M. Schuetter, M.D. Kasoji, A. Minard-Smith, C.A. Barden, et al., Short-read, high-throughput sequencing technology for STR genotyping, BioTechniques. 0 (2012) 1-6.

[22] Y. Yang, B. Xie, J. Yan, Application of next-generation sequencing technology in forensic science, Genomics Proteomics Bioinformatics. 12 (2014) 190-197.

[23] M.M. Holland, M.R. McQuillan, K.A. O'Hanlon, Second generation sequencing allows for mtDNA mixture deconvolution and high resolution detection of heteroplasmy, Croat.Med.J. 52 (2011) 299-313.

[24] R.S. Just, M.K. Scheible, S.A. Fast, K. Sturk-Andreaggi, J.L. Higginbotham, E.A. Lyons, et al., Development of forensic-quality full mtGenome haplotypes: success rates with low template specimens, Forensic.Sci.Int.Genet. 10 (2014) 73-79.

[25] E.A. Lyons, M.K. Scheible, K. Sturk-Andreaggi, J.A. Irwin, R.S. Just, A high-throughput Sanger strategy for human mitochondrial genome sequencing, BMC Genomics. 14 (2013) 881-2164-14-881.

[26] M.K. Gonder, H.M. Mortensen, F.A. Reed, A. de Sousa, S.A. Tishkoff, Whole-mtDNA genome sequence analysis of ancient African lineages, Mol.Biol.Evol. 24 (2007) 757-768.

[27] L. Fendt, B. Zimmermann, M. Daniaux, W. Parson, Sequencing strategy for the whole mitochondrial genome resulting in high quality sequences, BMC Genomics. 10 (2009) 139.

[28] Illumina, Human mtDNA Genome: For the Illumina Sequencing Platform, 2013. 
[29] Illumina, Nextera ${ }^{\circledR}$ XT DNA: Sample Preparation Guide, 2012.

[30] KAPA Biosystems, KAPA Library Quantification Kits for Illumina sequencing platforms, Version 4.11 .

[31] Illumina, MiSeq ${ }^{\circledR}$ System User Guide, 2013.

[32] S. Anderson, A.T. Bankier, B.G. Barrell, M.H. de Bruijn, A.R. Coulson, J. Drouin, et al., Sequence and organization of the human mitochondrial genome, Nature. 290 (1981) 457-465.

[33] R.M. Andrews, I. Kubacka, P.F. Chinnery, R.N. Lightowlers, D.M. Turnbull, N. Howell, Reanalysis and revision of the Cambridge reference sequence for human mitochondrial DNA, Nat.Genet. 23 (1999) 147.

[34] H.J. Bandelt, A. Kloss-Brandstatter, M.B. Richards, Y.G. Yao, I. Logan, The case for the continuing use of the revised Cambridge Reference Sequence (rCRS) and the standardization of notation in human mitochondrial DNA studies, J.Hum.Genet. 59 (2014) 66-77.

[35] K. Vancampenhout, B. Caljon, C. Spits, K. Stouffs, A. Jonckheere, L. De Meirleir, et al., A bumpy ride on the diagnostic bench of massive parallel sequencing, the case of the mitochondrial genome, PLoS One. 9 (2014) e112950.

[36] T. Maricic, M. Whitten, S. Paabo, Multiplexed DNA sequence capture of mitochondrial genomes using PCR products, PLoS One. 5 (2010) e14004.

[37] R.S. Just, J.A. Irwin, W. Parson, Mitochondrial DNA heteroplasmy in the emerging field of massively parallel sequencing, Forensic.Sci.Int.Genet. 18 (2015) 131-139.

[38] J.L. King, A. Sajantila, B. Budowle, mitoSAVE: mitochondrial sequence analysis of variants in Excel, Forensic.Sci.Int.Genet. 12 (2014) 122-125.

[39] M. Holland, J.A. McElhoe, A custom software solution for forensic mtDNA analysis of MiSeq data, Forensic Science International: Genetics Supplement Series. 5 (2015) e614.

[40] B. Rebolledo-Jaramillo, M.S. Su, N. Stoler, J.A. McElhoe, B. Dickins, D. Blankenberg, et al., Maternal age effect and severe germ-line bottleneck in the inheritance of human mitochondrial DNA, Proc.Natl.Acad.Sci.U.S.A. 111 (2014) 15474-15479. 
Tables and Figures

Table 1 . Sequencing quality metrics generated by the Sequencing Analysis Viewer (Illumina).

\begin{tabular}{|c|c|c|c|c|c|c|c|}
\hline $\begin{array}{c}\text { Sequencing } \\
\text { run }\end{array}$ & $\begin{array}{c}\text { Normalization } \\
\text { method }\end{array}$ & $\begin{array}{c}\text { MiSeq } \\
\text { instrument }\end{array}$ & $\begin{array}{c}\text { Cluster } \\
\text { density } \\
\left(\mathbf{k} / \mathbf{m m}^{\mathbf{2}}\right)\end{array}$ & $\begin{array}{c}\text { Clusters } \\
\text { passing } \\
\text { filter } \mathbf{\%})\end{array}$ & $\begin{array}{c}\text { Total } \\
\text { reads } \\
\text { passing } \\
\text { filter } \\
\text { (million) }\end{array}$ & $\begin{array}{c}\text { Median } \\
\mathbf{\text { Q30 }}\end{array}$ & $\begin{array}{c}\text { sample read } \\
\text { percentage }\end{array}$ \\
\hline Set 1 & $\begin{array}{c}\text { Concentration- } \\
\text { based (KAPA } \\
\text { qPCR) }\end{array}$ & AFDIL & 940 & 89.5 & 19.4 & 69.7 & $1.04 \%$ \\
\hline Set 2 & Bead-based & FBI & 953 & 91.7 & 20.8 & 67.4 & $1.03 \%$ \\
\hline
\end{tabular}




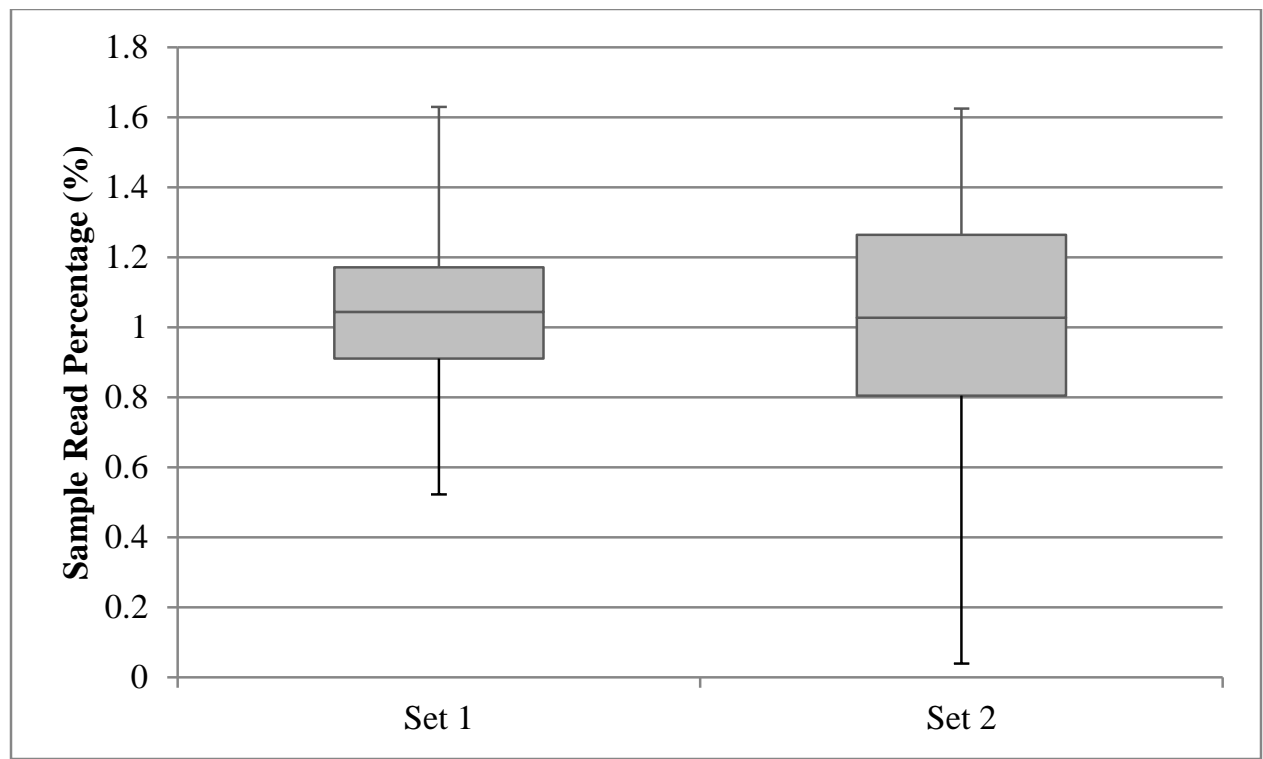

Figure 1. Box and whisker plot showing the distribution of sample read percentage.

Single column image 
Table 2. Concordance evaluation - comparison of NGS Set 1 to STS data.

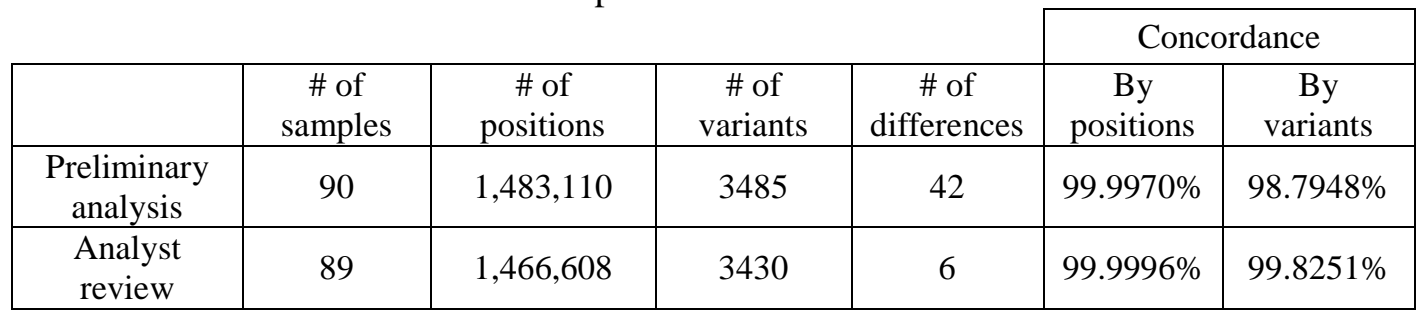


Table 3. Observed differences between NGS and STS data. Blank columns indicate a consistent call with STS and "--" indicates no variant call was made. Grayed out differences were not considered discordant and were excluded from the final concordance calculation. Variant calls inconsistent between NGS Sets 1 and 2 are in bold.

\begin{tabular}{|c|c|c|c|c|}
\hline Sample & STS Call & NGS Call - Set 1 & NGS Call - Set 2 & Reason for discordance \\
\hline mtGAfrLA0006 & $3594 \mathrm{~T}$ & & -- & Below coverage threshold \\
\hline mtGAfrGA0013 & $10873 \mathrm{C}$ & $10873 c$ & & \multirow{6}{*}{ Alignment artifact } \\
\hline mtGAfrOH0001 & $10873 \mathrm{C}$ & $10873 c$ & & \\
\hline mtGCaucMO0006 & $10873 \mathrm{C}$ & $10873 \mathrm{c}$ & & \\
\hline mtGHispCA0008 & $10873 \mathrm{C}$ & & $10873 \mathrm{c}$ & \\
\hline mtGHispCA0032 & $10873 \mathrm{C}$ & & $10873 \mathrm{c}$ & \\
\hline mtGHispNJ0005 & $10873 \mathrm{C}$ & $10873 c$ & & \\
\hline \multirow{28}{*}{ mtGAfrAL0004 } & $146 \mathrm{C}$ & $146 \mathrm{Y}$ & $146 \mathrm{Y}$ & \multirow{28}{*}{ Mixture } \\
\hline & -- & $769 \mathrm{R}$ & $769 \mathrm{R}$ & \\
\hline & -- & $825 \mathrm{~W}$ & $825 \mathrm{~W}$ & \\
\hline & $921 \mathrm{C}$ & $921 \mathrm{Y}$ & $921 \mathrm{Y}$ & \\
\hline & -- & $1018 \mathrm{R}$ & $1018 \mathrm{R}$ & \\
\hline & -- & & $2885 Y$ & \\
\hline & -- & $3666 \mathrm{R}$ & $3666 \mathrm{R}$ & \\
\hline & -- & & 3693R & \\
\hline & $5147 \mathrm{~A}$ & $5147 \mathrm{R}$ & $5147 \mathrm{R}$ & \\
\hline & $6446 \mathrm{~A}$ & $6446 \mathrm{R}$ & $6446 \mathrm{R}$ & \\
\hline & $6641 \mathrm{C}$ & $6641 \mathrm{Y}$ & $6641 \mathrm{Y}$ & \\
\hline & $6680 \mathrm{C}$ & $6680 \mathrm{Y}$ & & \\
\hline & -- & & $6989 R$ & \\
\hline & -- & 7146R & $7146 \mathrm{R}$ & \\
\hline & 7424G & $7424 \mathrm{R}$ & $7424 \mathrm{R}$ & \\
\hline & -- & 7521R & & \\
\hline & $8618 \mathrm{C}$ & $8618 \mathrm{Y}$ & $8618 \mathrm{Y}$ & \\
\hline & -- & $8655 \mathrm{Y}$ & & \\
\hline & -- & & 10688R & \\
\hline & -- & $13506 \mathrm{Y}$ & & \\
\hline & -- & $13650 \mathrm{Y}$ & $13650 \mathrm{Y}$ & \\
\hline & -- & & 13789Y & \\
\hline & -- & & 13880M & \\
\hline & $13886 \mathrm{C}$ & $13886 \mathrm{Y}$ & $13886 \mathrm{Y}$ & \\
\hline & -- & & 14178Y & \\
\hline & -- & $14203 R$ & $14203 R$ & \\
\hline & $14284 \mathrm{~T}$ & $14284 \mathrm{Y}$ & $14284 \mathrm{Y}$ & \\
\hline & -- & 14560R & & \\
\hline
\end{tabular}




\begin{tabular}{|c|c|c|c|c|}
\hline & -- & $14769 \mathrm{R}$ & $14769 \mathrm{R}$ & \\
\hline & -- & 15077R & & \\
\hline & $15301 \mathrm{~A}$ & $15301 \mathrm{R}$ & $15301 \mathrm{R}$ & \\
\hline & $15748 \mathrm{C}$ & $15748 Y$ & & \\
\hline & $16124 \mathrm{C}$ & $16124 \mathrm{Y}$ & $16124 \mathrm{Y}$ & \\
\hline & -- & $16126 \mathrm{Y}$ & & \\
\hline & $16171 \mathrm{G}$ & $16171 \mathrm{R}$ & $16171 \mathrm{R}$ & \\
\hline & $16256 \mathrm{~T}$ & $16256 \mathrm{Y}$ & $16256 \mathrm{Y}$ & \\
\hline & -- & $16293 \mathrm{R}$ & $16293 \mathrm{R}$ & \\
\hline & -- & $16311 \mathrm{Y}$ & $16311 Y$ & \\
\hline & - & $16519 \mathrm{Y}$ & $16519 \mathrm{Y}$ & \\
\hline mtGAfrFL0008 & $143 \mathrm{R}$ & $143 \mathrm{~A}$ & $143 \mathrm{~A}$ & \multirow{8}{*}{ Low level variant } \\
\hline mtGAfrNV0001 & -- & $8393 \mathrm{Y}$ & $8393 \mathrm{Y}$ & \\
\hline mtGAfrTX0005 & -- & $7270 Y$ & $7270 \mathrm{Y}$ & \\
\hline mtGAfrWI0001 & -- & & 9655R & \\
\hline mtGCaucIL0013 & -- & $6296 Y$ & $6296 Y$ & \\
\hline mtGCaucPA0010 & 189R & & -- & \\
\hline mtGHispCA0021 & $16263 \mathrm{C}$ & $16263 \mathrm{Y}$ & $16263 \mathrm{Y}$ & \\
\hline mtGHispHI0002 & -- & 9548R & 9548R & \\
\hline
\end{tabular}




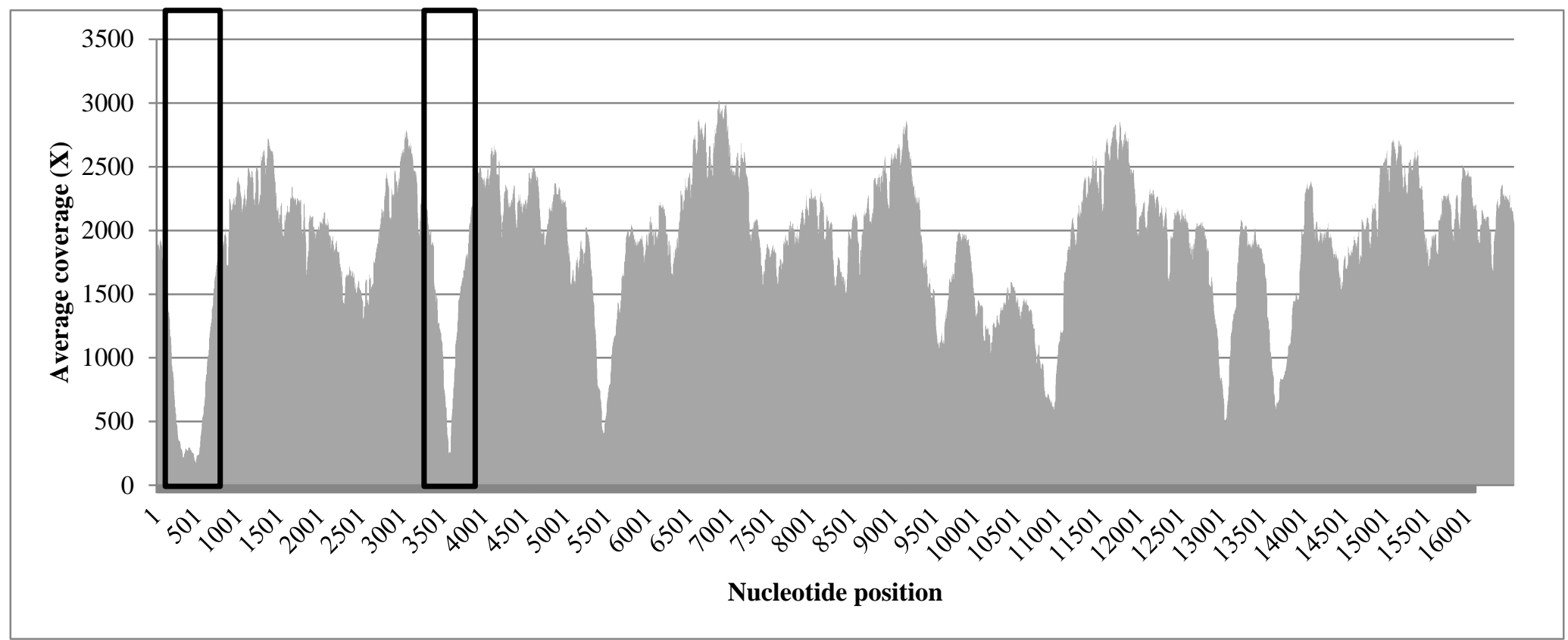

Figure 2. Coverage plot of average coverage (X) across the full mtGenome for both data sets. Black boxes indicated notable dips in coverage occurring in HVII and in the np 3500 region.

Two column image 
Table 4. Variant frequency average \pm standard deviation. Combined values were calculated across both data sets. Substitutions include np 10873 and indels include 3107del.

\begin{tabular}{|c|c|c|c|}
\hline & Set 1 & Set 2 & Combined \\
\hline Substitutions & $99.65 \% \pm 0.56$ & $99.64 \% \pm 0.58$ & $99.64 \% \pm 0.57$ \\
\hline Indels & $96.06 \% \pm 2.80$ & $96.13 \% \pm 1.96$ & $96.09 \% \pm 2.41$ \\
\hline PHPs & $41.60 \% \pm 37.55$ & $39.24 \% \pm 36.76$ & $40.43 \% \pm 37.03$ \\
\hline
\end{tabular}

